\title{
Stochastic Analysis of a Computer System with Unit Wise Cold Standby Redundancy and Failure of Service Facility
}

\author{
R. K. Yadav \\ Department of Statistics, \\ M. D. University, Rohtak - 124001, India. \\ E-mail: yadav.ramesh546@gmail.com \\ S. C. Malik \\ Department of Statistics, \\ M. D. University, Rohtak- 124001, India. \\ Corresponding author: sc_malik@rediffmail.com
}

(Received August 19, 2019; Accepted January 27, 2020)

\begin{abstract}
Here, we analyze stochastically a computer system by taking one more similar unit (called computer system) in cold standby redundancy. The computer system consists of hardware and software components together. The provision of a single service facility has been made for repairing hardware and up-grading the software. The failure of the service facility is considered which resumes the jobs with full efficiency as new after availing treatment. The failure rates of hardware and software components as well as failure rate of the service facility are taken as constant and thus follow negative exponential distribution. The treatment rate of the service facility, repair rate of the hardware and up-gradation rate of the software follow arbitrary distributions with different probability density functions. Efforts have been made to determine reliability measures in steady state by using SMP and RPT. The behavior of MTSF, availability and also the profit function is observed graphically for some particular situations of the parameters.
\end{abstract}

Keywords- Computer system, Unit wise redundancy, Failure of service facility, Reliability measures, Stochastic analysis, Profit analysis.

\section{Introduction}

In the era of modern technological world, the use of computer systems has increased extremely in almost all branches of sciences, engineering and public administration in order to complete the assignments in time and with perfectness. And, therefore it has become very difficult to ignore completely the usage of computer systems while fulfilling the job's requirements. The computer systems having these characteristics have possibility of the failures - hardware and software. These failures can cause a major setback to the owners in terms of financial loss, goodwill and loss of life. As a result of which the system becomes less reliable to the users. To deal with such situations the researchers have suggested several performance improvement techniques and thus reliability of computer systems. Over the years a good number of research papers have also been reported by the scholars on reliability modeling of redundant systems with different repair policies. The cold standby redundancy has been used frequently by the researchers by considering one or more unit. Malik and Sureria (2012) have studied computer systems with these concepts that is, by taking redundancy of the entire computer system in cold standby. Manglik and Ram (2013), Ram et al. (2013), Zhang (2015), Garg and Kadyan (2016) and Kumar and Goel (2016) discussed reliability models of cold standby systems with different repair strategies. However, these models have been analyzed under a common assumption that service facility remains busy in performing the assigned job continuously without any failure or difficulty. But, the failure of 
service facility cannot be ignored completely. The service facility may fail while performing the jobs due to carelessness on the part of the operator, unskilled knowledge about the system as well as may because of low quality components. Malik and Dhankhar (2011), Dhankhar et al. (2012), Bhardwaj and Singh (2015); Nandal and Rathee (2015) have considered the idea of failure of service facility (called server) while studying stochastic models of repairable systems. Further, Kumar and Saini (2018) have also studied computer systems with cold standby redundancy for the entire system by adopting different repair policies. However, the concept of failure of service facility has not been much introduced in the stochastic modeling of computer systems with unit wise redundancy in cold standby.

Hence, in this paper, the idea of unit wise cold standby redundancy is taken up during stochastic analysis of a computer system. The hardware and software components together form a computer system. The system model is developed by assuming failure of service facility with a constant failure rate. The service facility is subjected to failure, which resumes the jobs with full efficiency as new after availing treatment. The failure rates of hardware and software are taken as constant. The treatment rate of the service facility, repair rate of the hardware and up-gradation rate of the software follow arbitrary distributions with different probability density functions. The negative exponential distribution is taken up for the failure rate of the service facility. We determine some interesting reliability measures including MTSF, availability, busy periods, expected number of treatments and hardware repairs and software up-gradations in steady state by using SMP and RPT. The profit of the system model has been obtained under two policies. In first policy, the profit is evaluated by considering cost per unit time for busy period of the service facility and cost per unit time paid for treatment of the service facility. In second policy, the profit is evaluated by taking cost per unit hardware repair, per unit software up-gradation and cost per unit time paid for treatment of the service facility. The behavior of MTSF, availability and also the profit function has been observed graphically for some particular situations of the parameters. The comparison of the profit obtained under the policies has also been made graphically for particular values of the parameters.

\section{Notations and System Description}

\subsection{Notations}

The following notations have been used throughout the work:

$\begin{array}{ll}\text { MTSF } & \text { Mean Time to System Failure. } \\ \text { SMP } & \text { Semi-Markov Process. } \\ \text { RPT } & \text { Regenerative Point Technique. } \\ \text { MST } & \text { Mean Sojourn Time. } \\ \text { O/Cs } & \text { The unit is operative/ in cold standby. } \\ \text { a/b } & \text { Probability of hardware/software failure. } \\ \mathrm{x}_{1} / \mathrm{x}_{2} / \mu & \text { Hardware/software/ server failure rates. } \\ \text { HFUr/HFWr } & \text { The failed hardware is under/waiting for repair. } \\ \text { HFUR/HFWR } & \text { Failed hardware is continuously under/waiting for repair from prior state. } \\ \text { SFUg/SFWUg } & \text { The failed software is under/waiting for up-gradation. } \\ \text { SFUG/SFWUG } & \text { Software is continuously under/waiting for up-gradation from prior state. } \\ \text { SUt } & \text { The failed server (service facility) is under treatment. } \\ \text { SUT } & \text { The failed server (service facility) is continuously under treatment from prior state. } \\ \mathrm{h}(\mathrm{t}) / \mathrm{H}(\mathrm{t}) & \text { pdf/cdf of hardware repair time. } \\ \mathrm{u}(\mathrm{t}) / \mathrm{U}(\mathrm{t}) & \text { pdf/cdf of software repair time. } \\ \mathrm{s}(\mathrm{t}) / \mathrm{S}(\mathrm{t}) & \text { pdf/cdf of server treatment time. } \\ \mathrm{m}(\mathrm{t}) / \mathrm{M}(\mathrm{t}) & \text { pdf/cdf of hardware preventive maintenance time. }\end{array}$




$\mathrm{q}_{\mathrm{ij}}(\mathrm{t}) / \mathrm{Q}_{\mathrm{ij}}(\mathrm{t})$
$\mathrm{m}_{\mathrm{ij}}$
$\mathrm{M}_{\mathrm{i}}(\mathrm{t})$
$W_{i}^{H}(t)$
$W_{i}^{S}(t)$
$\mathrm{S} / \odot$
$* / *$
$P_{1}$
$P_{2}$
$C_{0}$
$C_{1} / C_{2}$
$C_{3} / C_{4}$
$C_{5}$

pdf/cdf of first passage time.

Contribution to $\operatorname{MST}\left(\mu_{\mathrm{i}}\right)$ in state $S_{\mathrm{i}}$ when system transits directly to state $S_{j}$.

Probability that the system up initially in regenerative state $S_{i}$ is up at time $t$ without visiting any other regenerative state.

Probability that the server is busy in the state $S_{i}$ due to hardware failure up to time ' $t$ ' without making any transition to any other regenerative state or returning to the same state via one or more non-regenerative states.

Probability that the server is busy in the state $S_{i}$ due to software up-gradation up to time ' $t$ ' without making any transition to any other regenerative state or returning to the same state via one or more non-regenerative states.

Standard notation for Laplace-Stieltjes convolution/Laplace convolution.

Symbol for Laplace Transform (LT)/Laplace Stieltjes Transform (LST).

Profit function by considering busy period cost of the server per unit time due to hardware repair/ software up-gradation and treatment cost of the server per unit time.

Profit function by taking Repair/up-gradation cost per unit time due to failure of hardware/software and treatment cost of the server per unit time.

System revenue per unit up-time.

Busy period cost of the server per unit time due to hardware repair/ software up-gradation.

Repair/up-gradation cost per unit time due to failure of hardware/software.

Treatment cost of the server per unit time.

\subsection{System Description}

We describe the following states of the system model:

$S_{0} \quad$ The Initial state in which system has one good unit (operative) and the other unit is in cold standby.

$S_{1} \quad$ In this state, one unit (entire computer system) is in operation and hardware component of the second unit is under repair.

$S_{2} \quad$ In this state, repair of hardware component of one unit is continued from prior state and hardware component of the second unit is waiting for repair.

$S_{3} \quad$ In this state, one unit is in operation and software component of the second unit is under up-gradation.

$S_{4} \quad$ In this state, server is failed and under treatment, one unit is operative and hardware component of the second unit is waiting for repair.

$S_{5} \quad$ In this state, server is failed and under treatment, waiting for repair of hardware component of one unit is continued from prior state and hardware component of the second unit is waiting for repair.

$S_{6} \quad$ In this state, treatment of the server is continued from prior state, waiting for repair of hardware component of one unit is continued from prior state and hardware component of second unit is waiting for repair.

$S_{7} \quad$ In this state, waiting for repair of hardware component of one unit is continued from prior state and hardware component of the second unit is under repair.

$S_{8} \quad$ In this state, treatment of the server is continued from prior state, waiting for repair of hardware component of one unit is continued from prior state and software component of the second unit is waiting for up-gradation.

$S_{9} \quad$ In this state, repair of hardware component of one unit is continued from prior state and software component of the second unit is waiting for up-gradation.

$S_{10} \quad$ In this state, up-gradation of software component of one unit is continued from prior state and software component of the second unit is waiting for up-gradation.

$S_{11} \quad$ In this state, server is failed and under treatment, hardware component of one unit is waiting for repair and waiting for up-gradation of software component of the second unit is continued from prior state.

$S_{12}$ In this state, hardware component of one unit is under repair and waiting for up-gradation of software component of the second unit is continued from prior state.

$S_{13} \quad$ In this state, hardware component of one unit is waiting for repair and up-gradation of software component of the second unit is continued from prior state. 
International Journal of Mathematical, Engineering and Management Sciences

Vol. 5, No. 3, 529-543, 2020

https://doi.org/10.33889/IJMEMS.2020.5.3.044

By using the notations and assumptions, the state transition diagram is developed for the system model as shown in Figure 1:

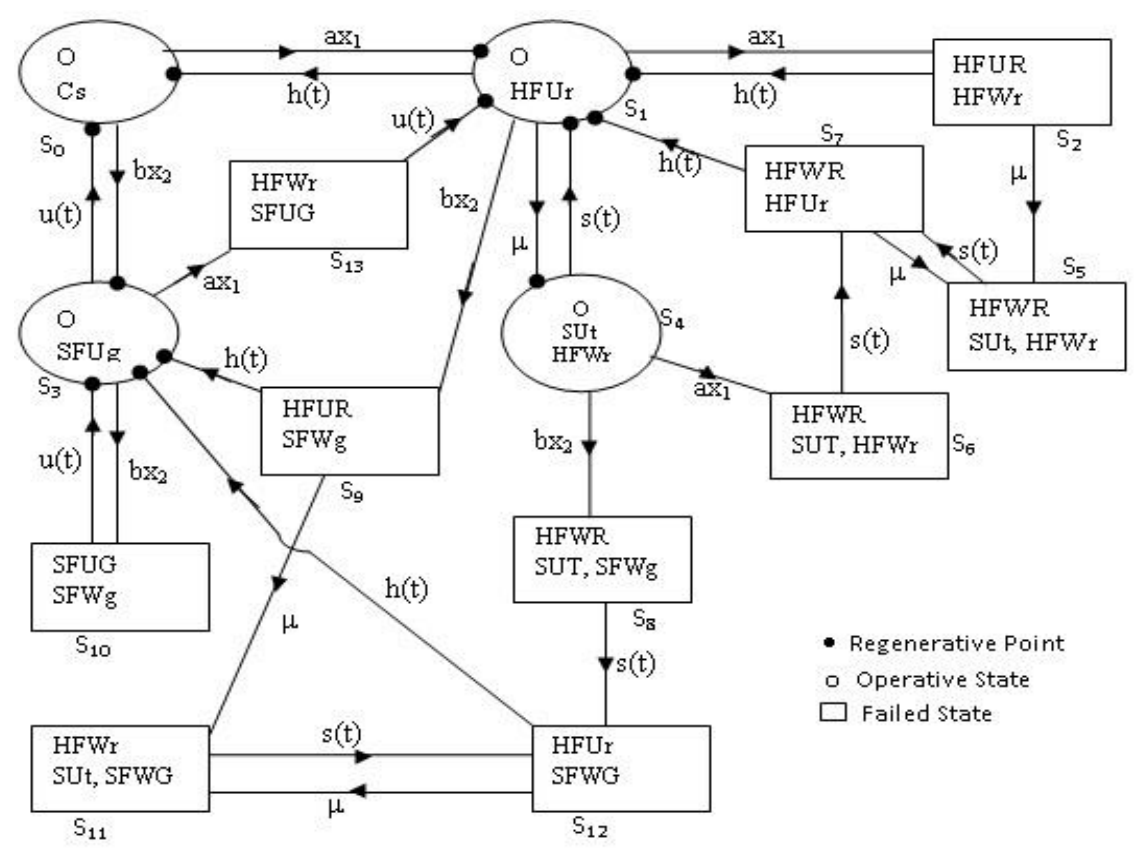

Figure 1. State transition diagram

\section{Reliability Measures}

\subsection{Transition Probabilities and Mean Sojourn Times}

We have the following expressions for non-zero elements that is, transition probabilities:

$p_{i j}=Q_{i j}(\infty)=\int_{0}^{\infty} q_{i j}(t) d t$ as

$p_{01}=\int_{0}^{\infty} a x_{1} e^{-\left(a x_{1}+b x_{2}\right) t} d t=\frac{a x_{1}}{a x_{1}+b x_{2}}$,

In the similar way, the following transition probabilities are calculated as

$$
\begin{aligned}
& p_{03}=\frac{b x_{2}}{a x_{1}+b x_{2}}, p_{10}=h^{*}\left(a x_{1}+b x_{2}+\mu\right), p_{12}=\frac{a x_{1}}{a x_{1}+b x_{2}+\mu}\left\{1-h^{*}\left(a x_{1}+b x_{2}+\mu\right)\right\}, \\
& p_{14}=\frac{\mu}{a x_{1}+b x_{2}+\mu}\left\{1-h^{*}\left(a x_{1}+b x_{2}+\mu\right)\right\}, \\
& p_{19}=\frac{b x_{2}}{a x_{1}+b x_{2}+\mu}\left\{1-h^{*}\left(a x_{1}+b x_{2}+\mu\right)\right\}, p_{21}=h^{*}(\mu), p_{25}=1-h^{*}(\mu), \\
& p_{30}=u^{*}\left(a x_{1}+b x_{2}\right), p_{3,10}=\frac{b x_{2}}{a x_{1}+b x_{2}}\left\{1-u^{*}\left(a x_{1}+b x_{2}\right)\right\}, \\
& p_{3,13}=\frac{a x_{1}}{a x_{1}+b x_{2}}\left\{1-u^{*}\left(a x_{1}+b x_{2}\right)\right\}, p_{41}=s^{*}\left(a x_{1}+b x_{2}\right), \\
& p_{46}=\frac{a x_{1}}{a x_{1}+b x_{2}}\left\{1-s^{*}\left(a x_{1}+b x_{2}\right)\right\}, p_{48}=\frac{b x_{2}}{a x_{1}+b x_{2}}\left\{1-s^{*}\left(a x_{1}+b x_{2}\right)\right\},
\end{aligned}
$$




$$
\begin{aligned}
& p_{57}=s^{*}(0)=p_{67}, p_{71}=h^{*}(\mu), p_{75}=1-h^{*}(\mu), p_{8,12}=s^{*}(0), p_{93}=h^{*}(\mu), p_{9.11}= \\
& 1-h^{*}(\mu), p_{10,3}=u^{*}(0), p_{11,12}=s^{*}(0), p_{12,3}=h^{*}(\mu), p_{12,11}=1-h^{*}(\mu), p_{13,1}= \\
& u^{*}(0), p_{11.2}=\frac{a x_{1}}{a x_{1}+b x_{2}+\mu}\left\{1-h^{*}\left(a x_{1}+b x_{2}+\mu\right)\right\} h^{*}(\mu), \\
& p_{13.9}=\frac{b x_{2}}{a x_{1}+b x_{2}+\mu}\left\{1-h^{*}\left(a x_{1}+b x_{2}+\mu\right)\right\} h^{*}(\mu) \text {, } \\
& p_{11.2(5,7)^{n}}=\frac{a x_{1}}{a x_{1}+b x_{2}+\mu}\left\{1-h^{*}\left(a x_{1}+b x_{2}+\mu\right)\right\}\left\{1-h^{*}(\mu)\right\}, \\
& p_{13.9(11,12)^{n}}=\frac{b x_{2}}{a x_{1}+b x_{2}+\mu}\left\{1-h^{*}\left(a x_{1}+b x_{2}+\mu\right)\right\}\left\{1-h^{*}(\mu)\right\}, \\
& p_{33.10}=\frac{b x_{2}}{a x_{1}+b x_{2}}\left\{1-u^{*}\left(a x_{1}+b x_{2}\right)\right\}, p_{31.13}=\frac{a x_{1}}{a x_{1}+b x_{2}}\left\{1-u^{*}\left(a x_{1}+b x_{2}\right)\right\}, \\
& p_{41.67}=\frac{a x_{1}}{a x_{1}+b x_{2}}\left\{1-s^{*}\left(a x_{1}+b x_{2}\right)\right\} h^{*}(\mu), \\
& p_{41.67(5,7)^{n}}=\frac{a x_{1}}{a x_{1}+b x_{2}}\left\{1-s^{*}\left(a x_{1}+b x_{2}\right)\right\}\left\{1-h^{*}(\mu)\right\}, \\
& p_{43.8,12}=\frac{b x_{2}}{a x_{1}+b x_{2}}\left\{1-s^{*}\left(a x_{1}+b x_{2}\right)\right\} h^{*}(\mu), \\
& p_{43.8,12(11,12)^{n}}=\frac{b x_{2}}{a x_{1}+b x_{2}}\left\{1-s^{*}\left(a x_{1}+b x_{2}\right)\right\}\left\{1-h^{*}(\mu)\right\} .
\end{aligned}
$$

We can prove that

$$
\begin{aligned}
& p_{01}+p_{03}=p_{10}+p_{12}+p_{14}+p_{19}=p_{21}+p_{25}=p_{30}+p_{3,10}+p_{3,13}=p_{41}+p_{46}+ \\
& p_{48}=1, \quad p_{71}+p_{75}=p_{93}+p_{9,11}=p_{12,3}+p_{12,11}=1, \quad p_{57}=p_{67}=p_{8,12}=p_{10,3}=
\end{aligned}
$$

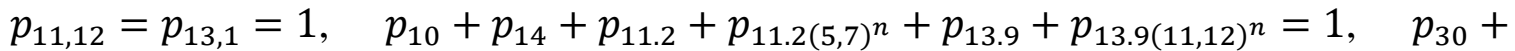

$$
\begin{aligned}
& p_{33.10}+p_{31.13}=1, p_{41}+p_{41.67}+p_{41.67(5,7)^{n}}+p_{43.8,12}+p_{43.8,12(11,12)^{n}}=1 \text {. }
\end{aligned}
$$

The MST $\left(\mu_{i}\right)$ in state $S_{\mathrm{i}}$ are calculated by the following relations

$$
\begin{aligned}
& m_{i j}=\left|-\frac{d}{d s} Q_{i j}^{* *}(s)\right|_{s=0} \text { and } \mu_{i}=\sum_{j} m_{i j} \text { where } Q_{i j}^{* *}(s) \text { is LST of } Q_{i j}(\mathrm{t}) \text {. Thus, we have } \\
& \mu_{0}=m_{01}+m_{03}, \quad \mu_{1}=m_{10}+m_{12}+m_{14}+m_{19}, \quad \mu_{2}=m_{21}+m_{25}, \quad \mu_{3}=m_{30}+ \\
& m_{3,10}+m_{3,13}, \mu_{4}=m_{41}+m_{46}+m_{48}, \mu_{5}=m_{57}, \mu_{6}=m_{67}, \mu_{7}=m_{71}+m_{75}, \mu_{8}= \\
& m_{8,12}, \quad \mu_{9}=m_{93}+m_{9,11}, \quad \mu_{10}=m_{10,3}, \quad \mu_{11}=m_{11,12}, \quad \mu_{12}=m_{12,3}+m_{12,11}, \\
& \mu_{13}=m_{13,1}, \quad \mu_{1}^{\prime}=m_{10}+m_{11.2}+m_{11.2(5,7)^{n}+m_{13.9}+m_{13.9(11,12)^{n}}+m_{14},} \\
& \mu_{3}^{\prime}=m_{30}+m_{33.10}+m_{31.13}, \quad \mu_{4}^{\prime}=m_{41}+m_{41.67}+m_{41.67(5,7)^{n}+m_{43.8,12}}+ \\
& m_{43.8,12(11,12)^{n} .}
\end{aligned}
$$


International Journal of Mathematical, Engineering and Management Sciences

Vol. 5, No. 3, 529-543, 2020

https://doi.org/10.33889/IJMEMS.2020.5.3.044

\subsection{Reliability and MTSF}

Let $\emptyset_{i}(t)$ be the c.d.f. of first passage time from regenerative state $S_{i}$ to a failed state. Regarding the failed state as absorbing state, we have following recursive relations for $\emptyset_{i}(t)$ :

$\emptyset_{i}(t)=\sum_{j} Q_{i j}(t)\left(S \emptyset_{j}(t)+\sum_{k} Q_{i k}(t)\right.$

where $j$ is an un-failed regenerative state to which the given regenerative state $i$ can transit and $k$ is a failed state to which the state $i$ can transit directly. Thus, the following equations are obtained as:

$$
\begin{aligned}
& \emptyset_{0}(t)=Q_{01}(t) \text { (S } \emptyset_{1}(t)+Q_{03}(t) \text { S } \emptyset_{3}(t), \\
& \emptyset_{1}(t)=Q_{10}(t) \text { (S } \emptyset_{0}(t)+Q_{12}(t)+Q_{14}(t) \text { (S } \emptyset_{4}(t)+Q_{19}(t), \\
& \emptyset_{3}(t)=Q_{30}(t) \text { S } \emptyset_{0}(t)+Q_{3,10}(t)+Q_{3,13}(t), \\
& \emptyset_{4}(t)=Q_{41}(t) \text { S } \emptyset_{1}(t)+Q_{46}(t)+Q_{48}(t) .
\end{aligned}
$$

Taking LST of above relation and solving for $\emptyset_{0}^{* *}(s)$ by Cramer's rule, we have

$$
R^{*}(s)=\frac{1-\emptyset_{0}^{* *}(s)}{s} \text {. }
$$

The Laplace Inverse Transform of $R^{*}(s)$ gives the reliability of the system. Also we have,

$$
\begin{aligned}
& \text { MTSF }=\lim _{s \rightarrow 0} \frac{1-\emptyset_{0}^{* *}(s)}{s}=\frac{N_{1}}{D_{1}}, \text { where } N_{1}=\left(1-p_{14} p_{41}\right)\left(p_{03} \mu_{3}+\mu_{0}\right)+p_{01}\left(p_{14} \mu_{4}+\mu_{1}\right) \\
& \text { and } D_{1}=\left(1-p_{14} p_{41}\right)\left(1-p_{03} p_{30}\right)-p_{01} p_{10} .
\end{aligned}
$$

\subsection{System Availability}

Let $A_{i}(t)$ be the probability that the system is in up-state at epoch ' $t$ ' given that the system entered regenerative state $S_{i}$ at $t=0$. The recursive relations for $A_{i}(t)$ are given as

$A_{i}(t)=M_{i}(t)+\sum_{j} q_{i j}^{(n)}(t) \mathbb{C} A_{j}(t)$

where $j$ is any successive regenerative state to which the regenerative state $i$ can transit through $n$ transitions. Thus, the following equations are obtained as:

$$
\begin{aligned}
& A_{0}(t)=M_{0}(t)+q_{01}(t)\left(A_{1}(t)+q_{03}(t) \subset A_{3}(t)\right. \text {, } \\
& A_{1}(t)=M_{1}(t)+q_{10}(t) \mathbb{C} A_{0}(t)+\left[q_{11.2}(t)+q_{11.2(5,7)^{n}}(t)\right] \mathbb{C} A_{1}(t)+\left[q_{13.9}(t)+\right.
\end{aligned}
$$

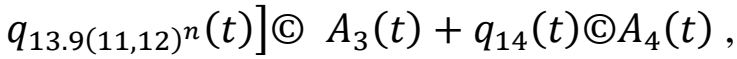

$$
\begin{aligned}
& A_{3}(t)=M_{3}(t)+q_{30}(t) \mathbb{C} A_{0}(t)+q_{31.13}(t)\left(\mathcal{C} A_{1}(t)+q_{33.10}(t) \mathbb{C} A_{3}(t)\right. \text {, } \\
& A_{4}(t)=M_{4}(t)+\left[q_{41}(t)+q_{41.67}(t)+q_{41.67(5,7)^{n}}(t)\right] \Subset A_{1}(t)+\left[q_{43.8,12}(t)+\right. \\
& \left.q_{43.8,12(11,12)^{n}}(t)\right] \Subset A_{3}(t) \text {. }
\end{aligned}
$$


where $M_{0}(t)=e^{-\left(a x_{1}+b x_{2}\right) t}, M_{1}(t)=e^{-\left(a x_{1}+b x_{2}+\mu\right) t} \bar{H}(t), M_{3}(t)=e^{-\left(a x_{1}+b x_{2}\right) t} \bar{U}(t)$, $M_{4}(t)=e^{-\left(a x_{1}+b x_{2}\right) t} \bar{S}(t)$.

We can obtain $A_{0}^{*}(s)$ by making use of LT technique and by using Cramer's rule.

The long run availability is given by

$$
\begin{aligned}
& A(\infty)=\lim _{s \rightarrow 0} s A_{0}^{*}(s)=\frac{N_{2}}{D_{2}} \text { where } N_{2}=p_{14} \mu_{4}+\mu_{1}+p_{10} \mu_{0} \text { and } \\
& D_{2}=p_{01}\left(p_{14} \mu_{4}^{\prime}+\mu_{1}^{\prime}\right)+\left(p_{19}+p_{14} p_{48}\right)\left(\mu_{3}^{\prime}+p_{30} \mu_{0}\right)+p_{10}\left[\mu_{0}\left(1-p_{3,10}\right)+\mu_{3}^{\prime} p_{03}\right]
\end{aligned}
$$

\subsection{Busy Period of Server}

The server can be busy due to hardware repair and software up-gradation.

\subsubsection{Due to Hardware Repair}

Let $B_{i}^{H}(t)$ be the probability that server is busy in repairing the unit due to hardware failure at epoch ' $t$ ' given that the system entered state $S_{i}$ at $t=0$. The recursive relations for $B_{i}^{H}(t)$ are given as

$$
B_{i}^{H}(t)=W_{i}^{H}(t)+\sum_{j} q_{i j}^{(n)}(t) \Subset B_{j}^{H}(t)
$$

where $j$ is any successive regenerative state to which the regenerative state $i$ can transit through $n$ transitions. Thus, the following equations are obtained as:

$$
\begin{aligned}
& B_{0}^{H}(t)=q_{01}(t)\left(B_{1}^{H}(t)+q_{03}(t) \mathbb{C} B_{3}^{H}(t),\right. \\
& B_{1}^{H}(t)=W_{1}^{H}(t)+q_{10}(t) \subseteq B_{0}^{H}(t)+\left[q_{11.2}(t)+q_{11.2(5,7)^{n}}(t)\right] \Subset B_{1}^{H}(t)+\left[q_{13.9}(t)+\right. \\
& \left.q_{13.9(11,12)^{n}}(t)\right] \mathbb{C} B_{3}^{H}(t)+q_{14}(t) \mathbb{C} B_{4}^{H}(t), \\
& B_{3}^{H}(t)=q_{30}(t) \mathbb{C} B_{0}^{H}(t)+q_{31.13}(t) \mathbb{C} B_{1}^{H}(t)++q_{33.10}(t) \mathbb{C} B_{3}^{H}(t), \\
& B_{4}^{H}(t)=\left[q_{41}(t)+q_{41.67}(t)+q_{41.67(5,7)^{n}}(t)\right] \Subset B_{1}^{H}(t)+\left[q_{43.8,12}(t)+\right.
\end{aligned}
$$

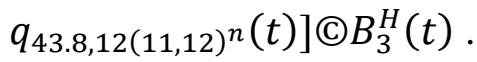

Where

$$
\left(b x_{2} e^{-\left(a x_{1}+b x_{2}+\mu\right) t}(C)\right] \bar{H}(t) \text {. }
$$

$$
W_{1}^{H}(t)=\left[e^{-\left(a x_{1}+b x_{2}+\mu\right) t}+\left(a x_{1} e^{-\left(a x_{1}+b x_{2}+\mu\right) t}(\mathcal{C} 1)+\right.\right.
$$

We can obtain $B_{0}^{H^{*}}(s)$ by making use of LT technique and by using Cramer's rule.

The busy time of server due to hardware repair is given by $B_{0}^{H}(\infty)=\lim _{s \rightarrow 0} s B_{0}^{H^{*}}(s)=\frac{N_{3}}{D_{2}}$, where $N_{3}=p_{01} W_{1}^{H^{*}}(0)$ and $D_{2}$ is already mentioned. 
International Journal of Mathematical, Engineering and Management Sciences

Vol. 5, No. 3, 529-543, 2020

https://doi.org/10.33889/IJMEMS.2020.5.3.044

\subsubsection{Due to Software Up-gradation}

Let $B_{i}^{S}(t)$ be the probability that server is busy in repairing the unit due to software up-gradation at epoch ' $t$ ' given that the system entered state $S_{i}$ at $t=0$. The recursive relations for $B_{i}^{S}(t)$ are given as:

$B_{i}^{S}(t)=W_{i}^{S}(t)+\sum_{j} q_{i j}^{(n)}(t) \Subset B_{j}^{S}(t)$

where $j$ is any successive regenerative state to which the regenerative state $i$ can transit through $n$ transitions. Thus, the following equations are obtained as:

$$
\begin{aligned}
& B_{0}^{S}(t)=q_{01}(t) @ B_{1}^{S}(t)+q_{03}(t) \Subset B_{3}^{S}(t),
\end{aligned}
$$

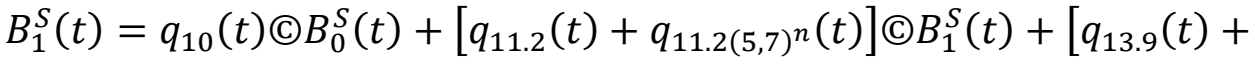

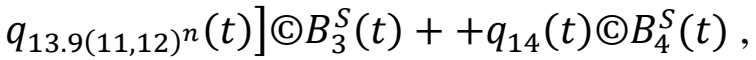

$$
\begin{aligned}
& B_{3}^{S}(t)=W_{3}^{S}(t)+q_{30}(t) \Subset B_{0}^{S}(t)+q_{31.13}(t) \Subset B_{1}^{S}(t)++q_{33.10}(t) \Subset B_{3}^{S}(t), \\
& B_{4}^{S}(t)=\left[q_{41}(t)+q_{41.67}(t)+q_{41.67(5,7)^{n}}(t)\right] \Subset B_{1}^{S}(t)+\left[q_{43.8,12}(t)+\right. \\
& \left.q_{43.8,12(11,12)^{n}}(t)\right] \Subset B_{3}^{S}(t) \text {, }
\end{aligned}
$$

where $W_{3}^{S}(t)=\left[e^{-\left(a x_{1}+b x_{2}\right) t}+\left(a x_{1} e^{-\left(a x_{1}+b x_{2}\right) t}(1)+\left(b x_{2} e^{-\left(a x_{1}+b x_{2}\right) t} @ 1\right)\right] \bar{U}(t)\right.$.

We can obtain $B_{0}^{S^{*}}(s)$ by making use of LT technique and by using Cramer's rule.

The busy time of server due to software up-gradation is given by

$B_{0}^{S}(\infty)=\lim _{s \rightarrow 0} B_{0}^{S^{*}}(s)=\frac{N_{4}}{D_{2}}$, where $N_{4}=W_{3}^{S^{*}}(0)\left[p_{19}+p_{14} p_{48}+p_{03} p_{10}\right]$ and $D_{2}$ is already mentioned.

\subsection{Expected Number of Hardware Repairs}

Let $R_{i}(t)$ be the expected number of the hardware repairs by the server in the interval $(0, \mathrm{t}]$ given that the system entered regenerative state $S_{i}$ at $t=0$. The expected number of the hardware repairs is given by

$R_{0}(\infty)=\lim _{s \rightarrow 0} s R_{0}^{* *}(s)$

The recursive relations for $R_{i}(t)$ are given as:

$R_{i}(t)=\sum_{j} Q_{i j}^{(n)}(t) \subseteq\left[\delta_{\mathrm{j}}+R_{j}(t)\right]$

where $j$ is any regenerative state to which the regenerative state $i$ can transit through $n$ transitions and $\delta_{\mathrm{j}}=1$, if $j$ is the regenerative state where server does job afresh, otherwise $\delta_{\mathrm{j}}=0$." Thus, the following equations are obtained as:

$$
R_{0}(t)=Q_{01}(t) \subseteq R_{1}(t)+Q_{03}(t) \subseteq R_{3}(t),
$$


International Journal of Mathematical, Engineering and Management Sciences

Vol. 5, No. 3, 529-543, 2020

https://doi.org/10.33889/IJMEMS.2020.5.3.044

$R_{1}(t)=Q_{10}(t) S\left[1+R_{0}(t)\right]+\left[Q_{11.2}(t)+Q_{11.2(5,7)^{n}}(t)\right] S\left[1+R_{1}(t)\right]+$

$\left[Q_{13.9}(t)+Q_{13.9(11,12)^{n}}(t)\right]$ S $\left[1+R_{3}(t)\right]+Q_{14}(t)\left(S R_{4}(t)\right.$,

$R_{3}(t)=Q_{30}(t)(S) R_{0}(t)+Q_{31.13}(t)(S) R_{1}(t)+Q_{33.10}(t)(S) R_{3}(t)$,

$R_{4}(t)=Q_{41}(t)(S) R_{1}(t)+\left[Q_{41.67}(t)+Q_{41.67(5,7)^{n}}(t)\right] S\left[1+R_{1}(t)\right]+\left[Q_{43.8,12}(t)+\right.$

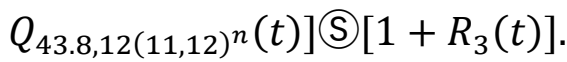

We can obtain $R_{0}^{* *}(s)$ by making use of LST technique and by using Cramer's rule.

The expected number of the hardware repairs is given by

$R_{0}(\infty)=\lim _{s \rightarrow 0} s R_{0}^{* *}(s)=\frac{N_{5}}{D_{2}}$ where $N_{5}=\left(1-p_{14} p_{41}\right) p_{01}$ and $D_{2}$ is already mentioned.

\subsection{Expected Number of Software Up-gradations}

Let $U_{i}(t)$ be the expected number of the software up-gradations by the server in the interval $(0, \mathrm{t}]$ given that the system entered regenerative state $S_{i}$ at $t=0$. The expected number of the software up-gradations is given by

$U_{0}(\infty)=\lim _{s \rightarrow 0} s U_{0}^{* *}(s)$

The recursive relations for $U_{i}(t)$ are given as:

$U_{i}(t)=\sum_{j} Q_{i j}^{(n)}(t) S\left[\delta_{j}+U_{j}(t)\right]$

where $j$ is any regenerative state to which the regenerative state $i$ can transit through $n$ transitions and $\delta_{\mathrm{j}}=1$, if $j$ is the regenerative state where server does job afresh, otherwise $\delta_{\mathrm{j}}=0$. Thus, the following equations are obtained as:

$$
\begin{aligned}
& U_{0}(t)=Q_{01}(t) \text { S } U_{1}(t)+Q_{03}(t) \text { S } U_{3}(t) \text {, } \\
& U_{1}(t)=Q_{10}(t) \text { S } U_{0}(t)+\left[Q_{11.2}(t)+Q_{11.2(5,7)^{n}}(t)\right] \text { S } U_{1}(t)+\left[Q_{13.9}(t)+\right.
\end{aligned}
$$

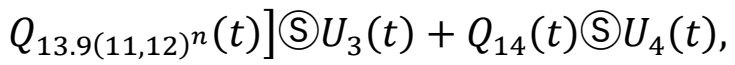

$$
\begin{aligned}
& U_{3}(t)=Q_{30}(t) \text { S }\left[1+U_{0}(t)\right]+Q_{31.13}(t) \text { S }\left[1+U_{1}(t)\right]+Q_{33.10}(t) \text { S }\left[1+U_{3}(t)\right] \text {, } \\
& U_{4}(t)=\left[Q_{41}(t)+Q_{41.67}(t)+Q_{41.67(5,7)^{n}}(t)\right] S U_{1}(t)+\left[Q_{43.8,12}(t)+\right.
\end{aligned}
$$

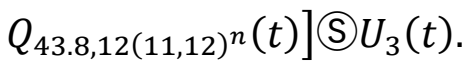

We can obtain $U_{0}^{* *}(s)$ by making use of LST technique and by using Cramer's rule.

The expected number of the software up-gradations is given by 
$U_{0}(\infty)=\lim _{s \rightarrow 0} U_{0}^{* *}(s)=\frac{N_{6}}{D_{2}} \quad$ where $\quad N_{6}=p_{19}+p_{14} p_{48}+p_{03} p_{10} \quad$ and $\quad D_{2} \quad$ is already mentioned.

\subsection{Expected Number of Treatments given to the Service Facility}

Let $T_{i}(t)$ be the expected number of the treatments given by the server in the interval $(0, \mathrm{t}]$ given that the system entered regenerative state $S_{i}$ at $t=0$. The expected number of the server treatments is given by

$T_{0}(\infty)=\lim _{s \rightarrow 0} s T_{0}^{* *}(s)$

The recursive relations for $T_{i}(t)$ are given as:

$T_{i}(t)=\sum_{j} Q_{i j}^{(n)}(t) S\left[\delta_{\mathrm{j}}+T_{j}(t)\right]$

where $j$ is any regenerative state to which the regenerative state $i$ can transit through $n$ transitions and $\delta_{\mathrm{j}}=1$, if $j$ is the regenerative state where server does job afresh, otherwise $\delta_{\mathrm{j}}=0$." Thus, the following equations are obtained as:

$$
\begin{aligned}
& T_{0}(t)=Q_{01}(t) \text { S } T_{1}(t)+Q_{03}(t)\left(\mathrm{S} T_{3}(t),\right. \\
& T_{1}(t)=Q_{10}(t) \text { S } T_{0}(t)+Q_{11.2}(t) S T_{1}(t)+Q_{11.2(5,7)^{n}}(t) \text { S }\left[1+T_{1}(t)\right]+Q_{13.9}(t) \text { S }
\end{aligned}
$$

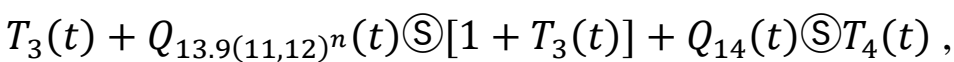

$$
\begin{aligned}
& T_{3}(t)=Q_{30}(t) \text { S } T_{0}(t)+Q_{31.13}(t) S T_{1}(t)+Q_{33.10}(t) S T_{3}(t) \\
& T_{4}(t)=\left[Q_{41}(t)+Q_{41.67}(t)+Q_{41.67(5,7)^{n}}(t)\right] \text { S }\left[1+T_{1}(t)\right]+\left[Q_{43.8,12}(t)+\right.
\end{aligned}
$$

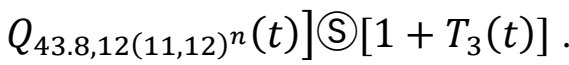

We can obtain $T_{0}^{* *}(s)$ by making use of LST technique and by using Cramer's rule.

The expected number of the treatments given to the server is given by

$T_{0}(\infty)=\lim _{s \rightarrow 0} s T_{0}^{* *}(s)=\frac{N_{7}}{D_{2}}$ where $N_{7}=\left[p_{25}\left(p_{12}+p_{19}\right)-p_{14}\right] p_{01}$ and $D_{2}$ is already mentioned.

\section{Profit Analysis}

In steady state, the profit of the system model can be obtained as

$$
\begin{aligned}
& P_{1}=C_{0} A_{0}(\infty)-C_{1} B_{0}^{H}(\infty)-C_{2} B_{0}^{S}(\infty)-C_{5} T_{0}(\infty) \text { and } \\
& P_{2}=C_{0} A_{0}(\infty)-C_{3} R_{0}(\infty)-C_{4} U_{0}(\infty)-C_{5} T_{0}(\infty) .
\end{aligned}
$$


International Journal of Mathematical, Engineering and Management Sciences

Vol. 5, No. 3, 529-543, 2020

https://doi.org/10.33889/IJMEMS.2020.5.3.044

\section{Graphical Presentation of Reliability Measures}

The values of the reliability measures and profit function are obtained by assuming negative exponential distribution for the random variables as: $h(t)=\alpha e^{-\alpha t}, u(t)=\beta e^{-\beta t}$ and $s(t)=\gamma e^{-\gamma t}$. The graphs for MTSF, availability and profit function are drawn for particular values of the parameters as shown respectively in the following figures:

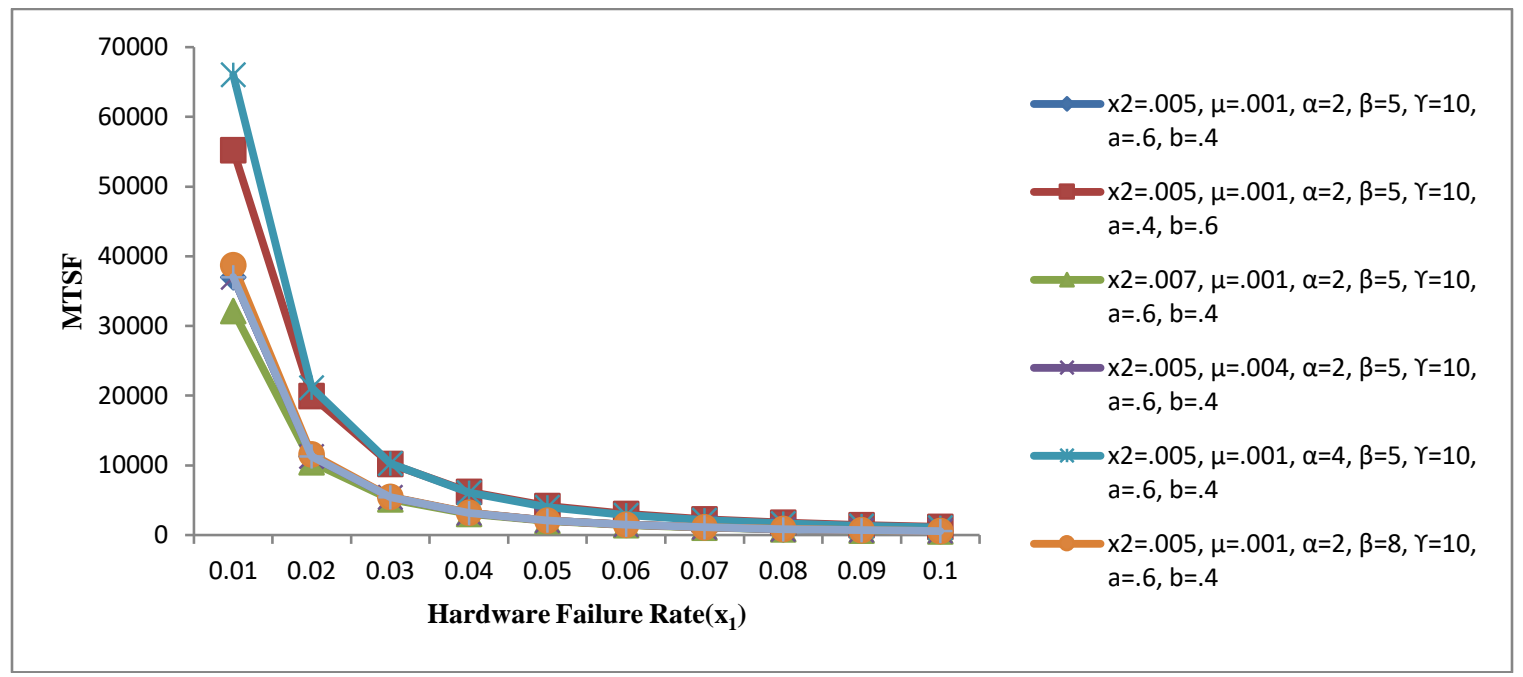

Figure 2. MTSF vs hardware failure rate $\left(x_{1}\right)$

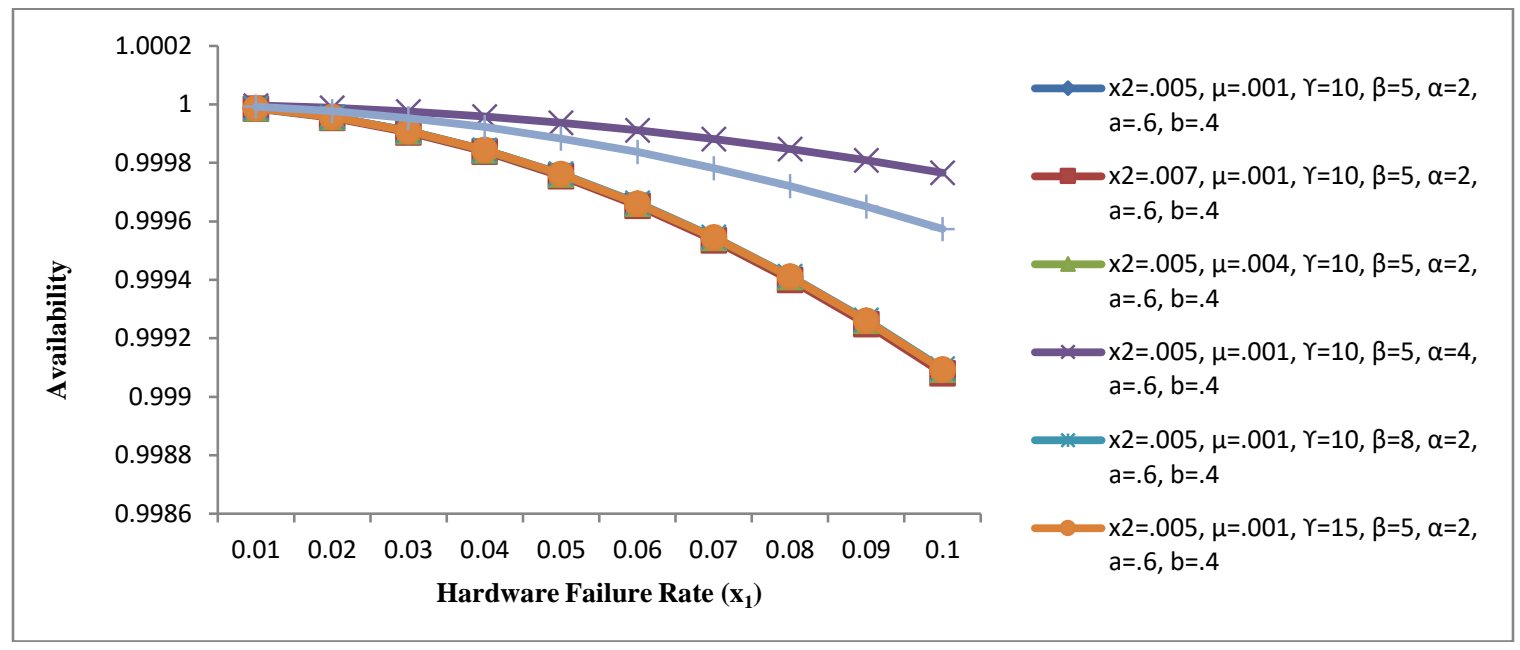

Figure 3. Availability vs hardware failure rate $\left(x_{1}\right)$ 
International Journal of Mathematical, Engineering and Management Sciences

Vol. 5, No. 3, 529-543, 2020

https://doi.org/10.33889/IJMEMS.2020.5.3.044

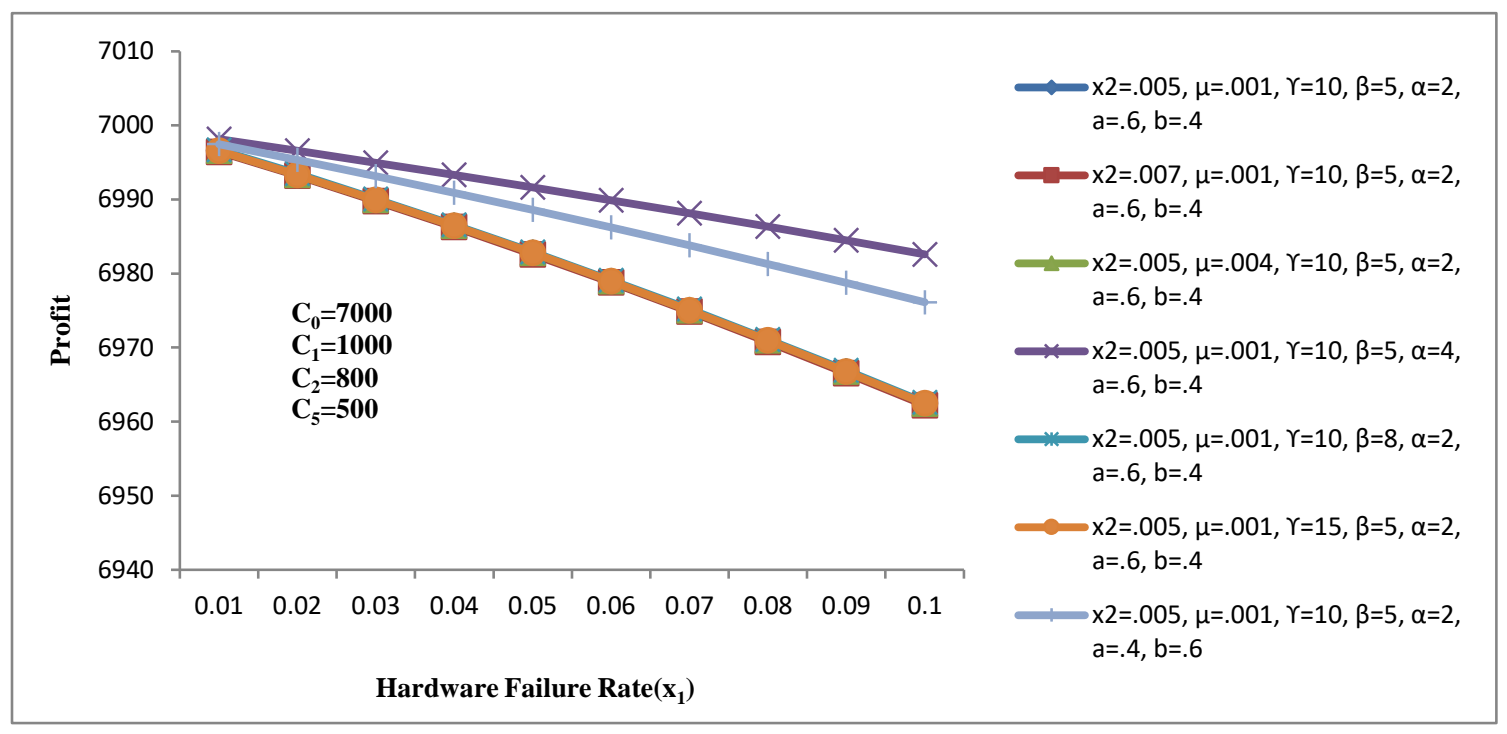

Figure 4. Profit $\left(\mathrm{P}_{1}\right)$ vs hardware failure rate $\left(x_{1}\right)$

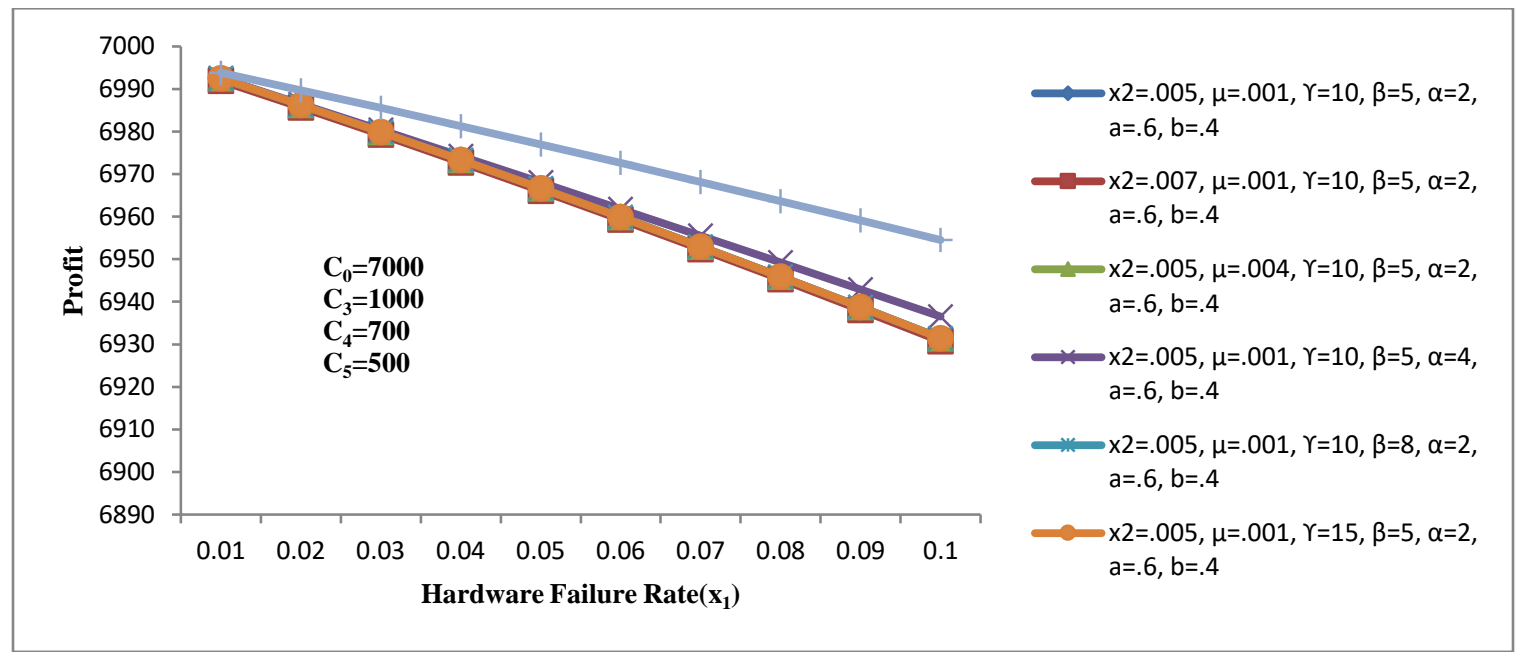

Figure 5. Profit $\left(\mathrm{P}_{2}\right)$ vs hardware failure rate $\left(x_{1}\right)$ 


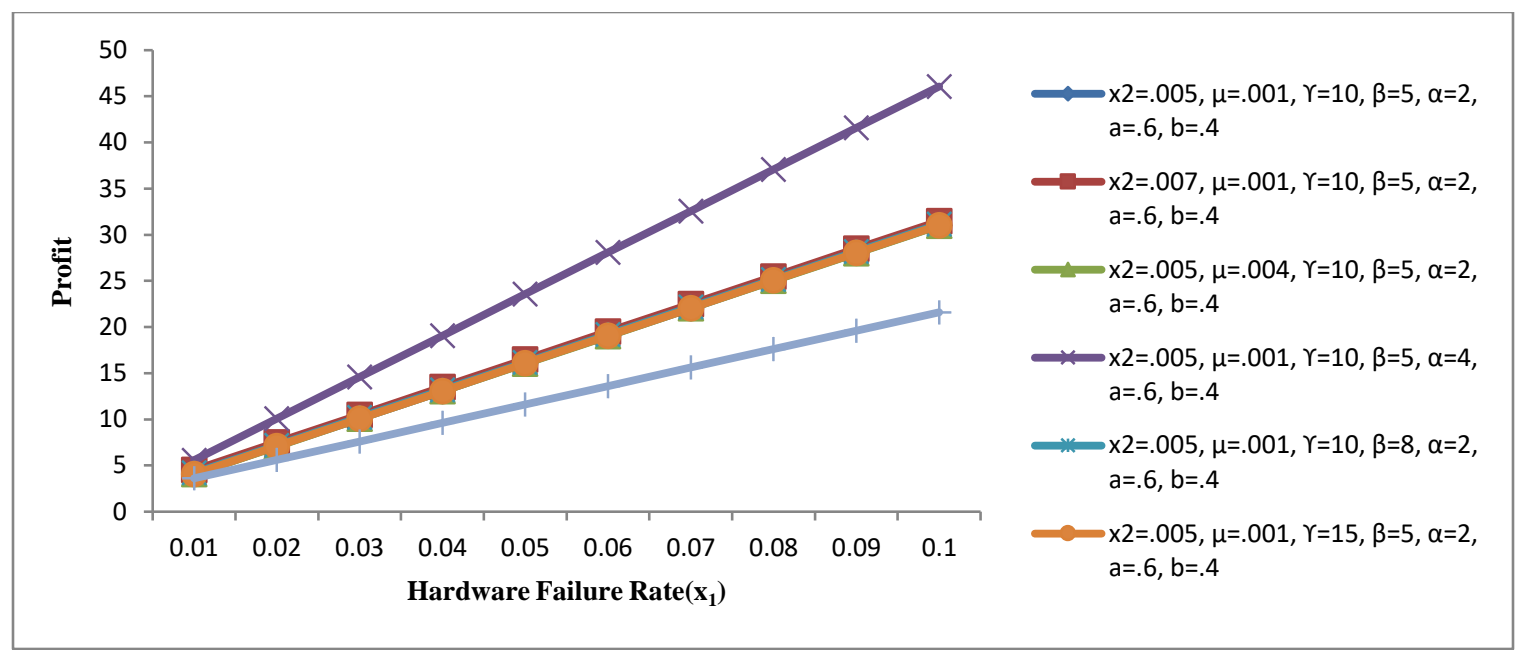

Figure 6. Profit $\left(\mathrm{P}_{1}-\mathrm{P}_{2}\right)$ vs hardware failure rate $\left(x_{1}\right)$

\section{Conclusion}

The stochastic analysis of a computer system has been carried out by taking one more unit (called computer system) in cold standby. The system model is developed by assuming failure of the service facility while performing jobs. The behavior of some important measures such as MTSF, availability and profit has been observed w.r.t. hardware failure rate $\left(\mathrm{x}_{1}\right)$ and for the fixed values of parameters associated with repair rate, up-gradation rate and treatment rate including $\mathrm{C}_{0}=7000$, $\mathrm{C}_{1}=1000, \mathrm{C}_{2}=800, \mathrm{C}_{3}=1000, \mathrm{C}_{4}=700, \mathrm{C}_{5}=500$, with $\mathrm{a}=0.6, \mathrm{~b}=0.4$ as shown respectively in Figures 2 to 6 . We found that MTSF, availability and profit function keep on decreasing with increase of $\mathrm{x}_{1}$ and $\mathrm{x}_{2}$ (failure rates) while they have improved values with the increase of hardware repair rate $(\alpha)$ and software up-gradation rate $(\beta)$ provided probability hardware failure is less than that of software failure. From Figure 6, we analyze that the profit of the system model under policy 1 is more than that of the profit obtained by considering policy 2 . Hence we can say that a computer system would be more profitable to use if costs are to be paid per unit time for busy period of the service facility rather than to pay the cost per unit hardware repair and software up-gradation.

\section{Conflict of Interest}

The work is original and has not been submitted anywhere for publication.

\section{Acknowledgement}

The authors sincerely appreciate the editor and reviewers for their time and valuable comments.

\section{References}

Bhardwaj, R.K., \& Singh, R. (2015). A cold-standby system with server failure and delayed treatment. International Journal of Computer Applications, 124(17), 31-36. 
International Journal of Mathematical, Engineering and Management Sciences

Vol. 5, No. 3, 529-543, 2020

https://doi.org/10.33889/IJMEMS.2020.5.3.044

Dhankar, A.K., Kumar, A., \& Malik, S.C. (2012). Cost-benefit analysis of a system with server failure and replacement of the unit at different failure modes subject to inspection. International Journal of Computer Applications, 47(1), 27-33.

Garg, V., \& Kadyan, M.S. (2016). Profit analysis of a two-unit cold standby system subject to preventive maintenance. International Journal of Statistics and Reliability Engineering, 3(1), 29-39.

Kumar, A., \& Saini, M. (2018). Profit analysis of a computer system with preventive maintenance and priority subject to maximum operation and repair times. Iran Journal of Computer Science, 1(3), 147153.

Kumar, J., \& Goel, M. (2016). Availability and profit analysis of a two unit cold standby system for general distribution. Cogent Mathematics, 3(1), 1-30.

Malik, S.C., \& Dhankar, A.K. (2011). Cost- benefit analysis of system reliability models with server failure during inspection and repair. International Journal of Statistics \& Analysis, 1(3), 265-278.

Manglik, M., \& Ram, M. (2013). Reliability analysis of a two unit cold standby system using Markov process. Journal of Reliability and Statistical Studies, 6(2), 65-80.

Nandal, J., \& Rathee, R. (2015). Stochastic analysis of a redundant system with server failure and conditional arrival time. International Journal of Statistics and Reliability Engineering, 2(1), 94-102.

Ram, M., Singh, S.B., \& Singh, V.V. (2013). Stochastic analysis of a standby system with waiting repair strategy. IEEE Transactions on Systems, Man, and Cybernetics: Systems, 43(3), 698-707.

Sureria, J.K., \& Malik, S.C. (2012). Probabilistic analysis of a computer system with arrival time of the server at $\mathrm{h} / \mathrm{w}$ failure and priority for replacement of the s/w over h/w repair. International Journal of Mathematics and Applied Statistics, 3(2), 115-128.

Zhang, X. (2015). Analysis of a cold standby repairable system with repairman extra work. Journal of System Science and Complexity, 28(5), 1015-1032.

Original content of this work is copyright (C) International Journal of Mathematical, Engineering and Management Sciences. Uses under the Creative Commons Attribution 4.0 International (CC BY 4.0) license at https://creativecommons.org/licenses/by/4.0/ 